\title{
Concatenated dynamical decoupling in a solid-state spin bath
}

\author{
W. M. Witzel and S. Das Sarma \\ Condensed Matter Theory Center, Department of Physics, \\ University of Maryland, College Park, MD 20742-4111
}

(Dated: November 7, 2018)

\begin{abstract}
Concatenated dynamical decoupling (CDD) pulse sequences hold much promise as a strategy to mitigate decoherence in quantum information processing. It is important to investigate the actual performance of these dynamical decoupling strategies in real systems that are promising qubit candidates. In this Rapid Communication, we compute the echo decay of concatenations of the Hahn echo sequence for a solid-state electronic spin qubit in a nuclear spin bath using a cluster expansion technique. We find that each level of concatenation reverses the effect of successive levels of intrabath fluctuations. On the one hand, this advances CDD as a versatile and realistic decoupling strategy. On the other hand, this invalidates, as overly optimistic, results of the simple pair approximation used previously to study restoration, through CDD, of coherence lost to a mesoscopic spin bath.
\end{abstract}

PACS numbers: 03.65.Yz; 03.67.Lx, 76.60.Lz

Successful large-scale quantum computation which relies exlusively on quantum error correcting codes necessitates extremely weak quantum decoherence $\left(<10^{-4}\right)$; this is difficult, if not impossible, to achieve in the strongly coupled solid-state environment. This stringent requirement arises from the fact that the quantum error correction (QEC) overhead grows exponentially (e.g., the number of auxiliary qubits needed for encoding) with the magnitude of decoherence. At a local level, decoherence can be mitigated by logically encoding qubits in a subspace that is less sensitive to noise and by applying dynamical decoupling (DD) controls. To meet QEC requirements, it is likely necessary to combine logical qubit encoding and DD in solid-state systems $\stackrel{\underline{1}}{\underline{1}}$ We focus our study on DD, which may employ deterministic, 2.3 .4 random,,$\frac{5}{\underline{a}}$ or hybrid deterministic-random ${ }^{6}$ sequence of qubit controls to partially reverse the effects of decoherence. Deterministic sequences hold promise for slow, predictable bath systems such as the nuclear spin bath that we consider. In a universal DD sequence, the timeaveraged Hamiltonian decouples the system from the bath, destroying the lowest-order system-bath coupling in a Magnus ${ }^{\underline{\underline{T}}}$ expansion $\underline{\underline{4}}$ The Hahn echo, with a single $\pi$ pulse applied midway through the evolution, is a simple universal DD sequence for a dephasing qubit. Periodic $\mathrm{DD}$ (PDD), repetitive applications of universal DD proven effective for nuclear magnetic resonance purposes for many years, $\underline{\underline{8}}$ simply trades an increase in the number of applied pulses for an increase in their frequency. Higher orders of the Magnus expansion, however, limit the performance of PDD; it is possible to overcome this effect using concatenated DD (CDD), recursive applications of a universal DD sequence $\underline{\underline{4}}$

In this Rapid Communication, we consider an archetypical qubit, a localized electron spin in a semiconductor at low temperature. The energy relaxation process of this spin can be made arbitrarily slow by lowering the temperature (e.g., $T_{1} \gg 1 \mathrm{~ms}$ for $T \sim 100 \mathrm{mK}$ ) because phonons are exponentially suppressed below the
Bloch-Gruneisen temperature $(\sim 10 \mathrm{~K})$. In the limit of a strong applied magnetic field, slow fluctuations of the surrounding nuclear spins will induce electron spin dephasing ("spectral diffusion" $\underline{\underline{9}}$ ) as the primary decoherence mechanism on a time scale of $T_{2} \sim 1 \mu \mathrm{s}$ (GaAs) $100 \mu \mathrm{s}(\mathrm{Si}: \mathrm{P})$. We show that this decoherence can be corrected, in principle, with arbitrary precision by using concatenations of the Hahn echo. The true reason for this, interestingly, goes beyond consideration of orders in the Magnus expansion; each concatenation of the sequence reverses the effect of successive levels of intrabath processes (specifically, CDD cancels orders of an intrabath perturbation).

There have been general theoretical discussions $\frac{4}{\underline{4}}$ about using CDD for qubit preservation without quantitative applications to specific realistic models of qubits in mesoscopic baths. To the extent that specific mesoscopic applications $\frac{10}{\underline{a}}$ exist for the spin qubit example considered in this paper, they invariably involve highly simplified and uncontrolled (as well as untested) models, e.g., the nuclear spin pair approximation, which fails for the realistic spin bath in the context of CDD. The pair approximation fails precisely because CDD, beyond canceling orders of a time (effectively Magnus) expansion, reverses the effect of successive levels of intrabath processes. As these lower levels of interactions are reversed (e.g., a single flip-flop of a pair of nuclei), larger clusters become perturbative equals to pairs and can dominate decoherence when they are greater in number. These larger clusters will diminish the effect of CDD (Refs. 10 give overly optimistic predictions). Nonetheless, we show that, contrary to PDD, concatenation allows one to obtain the same fidelity while decreasing the frequency of applied pulses. Our work is, to the best of our knowledge, the first theoretical application of the CDD scheme for a realistic situation, establishing, as a matter of principle, the reasonable prospects for perpetual coherent control of a qubit. In proof of concept, we demonstrate this in the form of quantum memory retention; however, it is 
known ${ }^{2}$ to be possible to effect nontrivial quantum evolution while performing DD. In fact, with proper logic encoding such that logical and DD operations commute, it may be possible 11 to perform CDD and quantum logic simultaneously, making CDD a viable strategy to meet the error correction threshold for a fault-tolerant solidstate spin quantum computer.

The continuous electron spin dephasing due to its coupling to the nuclear spin bath is a difficult process to ana-

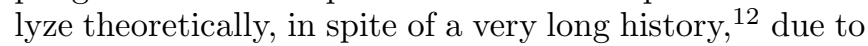
the quantum, non-Markovian nature of nuclear spin flipflops. We previously 13,14 developed a quantum theory of nuclear-induced spectral diffusion using a formally exact cluster expansion technique and analyzed the Hahn echo decay of solid-state spin qubits, and later $\frac{15}{15}$ adapted the technique to study the periodic Carr-Purcell-MeiboomGill 8 (CPMG) sequence. Concatenations of the Hahn echo sequence were analyzed in Refs. 10 for mesoscopic quantum-dot baths, which, however, employed a pair approximation that is not accurate in light of the perturbative cancellations that CDD sequences induce.

Specifically, we treat a donor electron spin in Si:P and a quantum dot electron spin in GaAs. Our electron spin qubit will precess in an applied magnetic field $(\sim 1 \mathrm{~T})$ at its Zeeman frequency $(\sim 10 \mathrm{GHz})$ with some fluctuations due to hyperfine (hf) interactions $(\sim 100 \mathrm{kHz})$ with a nuclear spin bath. The strong electron Zeeman energies compared with hf and nuclear Zeeman energies $(\sim 10 \mathrm{MHz})$ suppresses direct hf interactions that would flip the electron spin. Most importantly, the nuclei also interact with each other magnetically through their dipoles $(\sim 10 \mathrm{~Hz})$ which, if unchecked, will lead to an irreversible loss of information.

A general dephasing Hamiltonian may be written in the form $\hat{\mathcal{H}}=\sum_{ \pm}| \pm\rangle \hat{\mathcal{H}}_{ \pm}\langle \pm|$, where $\hat{\mathcal{H}}_{ \pm}$acts only upon the bath's Hilbert space. We can split $\hat{\mathcal{H}}_{ \pm}$into qubitdependent and -independent parts, so that $\hat{\mathcal{H}}_{ \pm}= \pm \hat{\mathcal{H}}_{q b}+$ $\epsilon \hat{\mathcal{H}}_{b b}$, where $\hat{\mathcal{H}}_{q b}$ contains the qubit-bath interaction terms and $\hat{\mathcal{H}}_{b b}$ contains the intrabath interaction terms. The $\epsilon$ is a bookkeeping parameter that will be discussed later in terms of an intrabath perturbation. From the Fermi-contact hf interaction, $\hat{H}_{q b}=\frac{1}{2} \sum_{n} A_{n} \hat{I}_{n z}$, where $A_{n}$ is proportional to the probability of the electron being at the $n$th nuclear site. From the secular dipolar interaction, $\hat{\mathcal{H}}_{b b}=\sum_{n \neq m}^{\prime} b_{n m} I_{n+} I_{m-}-\sum_{n \neq m} 2 b_{n m} I_{n z} I_{m z}$ where the first summation is restricted to pairs of like nuclei (so that Zeeman energy is preserved when they flip-flop) and $b_{n m}$ are dipolar coupling constants. We have included the most relevant interactions in the limit of a strong applied field as discussed in detail in Ref. 14 .

Given an $\hat{\mathcal{H}}=\sum_{ \pm}| \pm\rangle \hat{\mathcal{H}}_{ \pm}\langle \pm|$, the evolution operator $\hat{U}$ for any sequence of $\pi$ pulses (acting perpendicularly to the applied field) may be written in the form $\hat{U}=\sum_{ \pm}| \pm\rangle \hat{U}^{ \pm}\langle \pm|\left(\right.$or $\left.\hat{U}=\sum_{ \pm}|\mp\rangle \hat{U}^{ \pm}\langle \pm|\right)$for an even (or odd) number of applied pulses. With the up-down free evolution operators denoted $\hat{U}_{0}^{ \pm}(\tau)=\exp \left(i \hat{\mathcal{H}}_{ \pm} \tau\right)$, the Hahn echo sequence of $\tau \rightarrow \pi \rightarrow \tau \rightarrow \pi$, with an extra $\pi$ pulse at the end to return the electron spin to its original orientation, has up-down evolution operators of $\hat{U}_{1}^{ \pm}=\hat{U}_{0}^{\mp}(\tau) \hat{U}_{0}^{ \pm}(\tau)$. This trivially modified Hahn echo is the first level of the CDD series we consider. The Hahn echo sequence is best known as a means $\frac{12}{2}$ to eliminate the effects of inhomogeneous broadening (reversing the effects of a distribution of electron Zeeman frequencies in an ensemble). However, the Hahn echo is also an elementary DD sequence because the time-averaged Hamiltonian, proportional to $\hat{\mathcal{H}}_{+}+\hat{\mathcal{H}}_{-} \propto \hat{\mathcal{H}}_{b b}$, involves no qubit-bath interactions (in this respect, it does not yield a proper $T_{2}$ time consistent with the free induction decay of a single qubit $\frac{16}{6}$ ). As a DD sequence, it may be concatenated in hopes of successively improving qubit preservation times. $\stackrel{\underline{4}}{=}$ At the $l$ th level, our CDD pulse sequence for $l>0$ is $\mathrm{p}_{l}:=\mathrm{p}_{l-1} \rightarrow \pi \rightarrow \mathrm{p}_{l-1} \rightarrow \pi$, and $\mathrm{p}_{0}:=\tau$. With each concatenation, we do to the previous sequence what the Hahn echo does to free evolution and in this way obtain improved DD. This sequence may be simplified by noting that two $\pi$ pulses in sequence do nothing. Thus,

$$
\mathrm{p}_{l}:=\left\{\begin{array}{ll}
\mathrm{p}_{l-1} \rightarrow \pi \rightarrow \mathrm{p}_{l-1} & , \text { odd } l \\
\mathrm{p}_{l-1} \rightarrow \mathrm{p}_{l-1} & \text { even } l
\end{array},\right.
$$

and the up-down evolution operators at level $l$ have the recursive form of 10

$$
\hat{U}_{l}^{ \pm}=\hat{U}_{l-1}^{\mp} \hat{U}_{l-1}^{ \pm} .
$$

In order to characterize the coherence decay, we consider the transverse component of the qubit's expectation value; normalized to a maximum of 1 , the pulse sequence echo $v_{E}$ is defined in this way such that $v_{E}=$ $\left\|\left\langle\left[\hat{U}^{-}\right]^{\dagger} \hat{U}^{+}\right\rangle\right\|=\|\langle\hat{W}\rangle\|$ where $\hat{W} \equiv\left[\hat{U}^{-}\right]^{\dagger} \hat{U}^{+}$and the $\langle\ldots\rangle$ denotes an appropriately weighted average over the bath states. We assume that the bath is fully random and use equal weights in averaging over bath states $\frac{17}{17}$

We can solve this mesoscopic quantum problem using a cluster method $\stackrel{13,14}{14}$ Consider expanding $\hat{W}$ such that $\hat{W}=\sum_{n=0}^{N} \hat{W}^{[n]}$, where $\hat{W}^{[n]}$ contains contributions to $\hat{W}$ that involve $n$ separate clusters of "operatively" interacting nuclei. To be specific, the set of nuclei involved in a term of $\hat{W}^{[1]}$ must all be connected together via factors of bilinear interaction operators to form a single connected cluster. Clusters have spatial proximity when interactions are local. If it is possible to approximate $\left\langle\hat{W}^{[1]}\right\rangle$ by including only clusters up to some small size that is much less than the number of nuclei in the bath, $N$, and if the bath is effectively uncorrelated initially (e.g., a random bath), then $\left\langle\hat{W}^{[n]}\right\rangle \approx\left\langle\hat{W}^{[1]}\right\rangle^{n} / n$ !. In this "cluster approximation,"

$$
v_{E}=\|\langle\hat{W}\rangle\| \approx \exp \left(\operatorname{Re}\left\{\left\langle\hat{W}^{[1]}\right\rangle\right\}\right) .
$$

This approximation can be tested by quantifying the contributions of extraneous terms due to overlapping clusters when distributing through $\left\langle\hat{W}^{[1]}\right\rangle^{n} \stackrel{14}{=}$ Formally, $\left\langle\hat{W}^{[1]}\right\rangle$ is the sum of all cluster contributions, $\left\langle\hat{W}^{[1]}\right\rangle=\sum_{\mathcal{C}}\left\langle\hat{W}_{\mathcal{C}}^{\prime}\right\rangle$, 


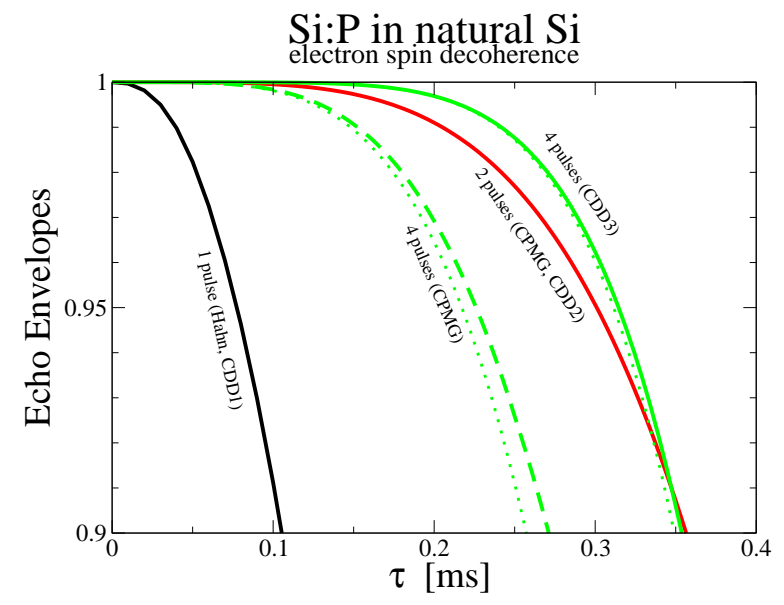

FIG. 1: Echo decay of an electron bound to a $\mathrm{P}$ donor in natural Si with an applied magnetic field along the [100] lattice direction for different DD pulse sequences as a function of the minimum time $\tau$ between consecutive pulses. The dotted lines give corresponding results to the lowest order in the intrabath perturbation. Only the first $90 \%$ of the decay is shown to avoid displaying regions in which the cluster expansion fails to converge. Conveniently, coherence much better than $90 \%$ is necessary for QEC protocols and the long-time coherence decay is irrelevant.

where the contribution from some cluster $\mathcal{C}$ may be computed using the recursive formula

$$
\left\langle\hat{W}_{\mathcal{C}}^{\prime}\right\rangle=\left\langle\hat{W}_{\mathcal{C}}\right\rangle-\sum_{\substack{\left\{\mathcal{C}_{i}\right\} \text { disjoint, } \\ \mathcal{C}_{i} \neq \emptyset, \mathcal{C}_{i} \subset \mathcal{C}}} \prod_{i}\left\langle\hat{W}_{\mathcal{C}_{i}}^{\prime}\right\rangle,
$$

and $\left\langle\hat{W}_{\mathcal{C}}\right\rangle$ is simply the solution to $\langle\hat{W}\rangle$ including only the nuclei in the set $\mathcal{C}$. The cluster expansion results from expanding $\left\langle\hat{W}^{[1]}\right\rangle$ in Eq. (33) to include clusters of successively increasing size.

We make a comparison between our convergent cluster expansion results for CDD and earlier calculated 15 CPMG pulse sequence echoes of both an electron bound to a $\mathrm{P}$ donor in natural Si (Fig. (1) and a quantum dot electron in GaAs (Fig. 2) plotted as a function of the minimum time $\tau$ between consecutive pulses. The twopulse CPMG sequence is the same as the CDD2 sequence $(l=2 \mathrm{CDD})$, and longer even-pulsed CPMG sequences are simple repetitions of the CDD2 sequence. The notable improvement of even-pulsed CPMG sequences over the Hahn echo $\frac{15}{15}$ is directly due to its extra level of concatenation. With an increasing number of CPMG pulses, the coherence as a function of $\tau$ is diminished, but, as noted in Ref. 15, the coherence as a function of the total sequence time, $t=4 \nu \tau$ for a sequence with $2 \nu$ pulses, tends to improve. With each level of concatenation in the CDD series, short-time coherence as a function of either $\tau$ or the total sequence time $t=2^{l+1} \tau$ improves. This demonstrates the restorative power of concatenation.

We can understand the reason for CDD coherence

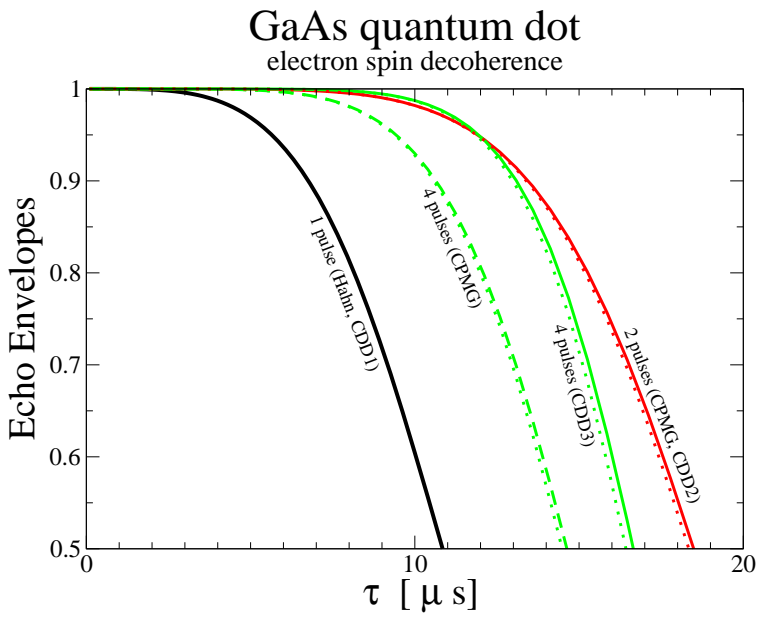

FIG. 2: Echo decay of a quantum dot (Fock-Darwin radius of $\ell=25 \mathrm{~nm}$ and quantum well thickness of $z_{0}=8.5 \mathrm{~nm}$ ) electron in GaAs with an applied magnetic field along the [110] lattice direction for different DD pulse sequences as a function of the minimum time $\tau$ between consecutive pulses. The dotted lines give corresponding results to the lowest order in the intrabath perturbation.

restoration as successive cancellations in perturbative orders. In a perturbation whose order increases with increasing cluster size, the lowest order of $\langle\hat{W}\rangle-1$ is equivalent to the same order of $\left\langle\hat{W}^{[1]}\right\rangle$ because terms of $\hat{W}$ with multiple clusters are automatically higher-order terms (being products of lower-order terms). This is formal equivalence that we use for convenience only (the entire purpose of the cluster expansion is to avoid applying such a perturbative expansion to $\langle\hat{W}\rangle$ directly because it would fail to converge due to the large size of the bath).

Using the unitarity property of the evolution operators in $\hat{W}_{l}=\left[\hat{U}_{l}^{-}\right]^{\dagger} \hat{U}_{l}^{+}$and defining $\Delta_{l} \equiv \hat{U}_{l}^{+}-\hat{U}_{l}^{-}$,

$$
\operatorname{Re}\left\{\left\langle\hat{W}_{l}\right\rangle\right\}=1-\frac{1}{2}\left\langle\Delta_{l}^{\dagger} \Delta_{l}\right\rangle
$$

so that $\operatorname{Re}\left\{\left\langle\hat{W}_{l}\right\rangle\right\}-1=-\mathcal{O}\left(\Delta_{l}^{2}\right)=\operatorname{Re}\left\{\left\langle\hat{W}_{l}^{[1]}\right\rangle\right\}$ and $v_{E}=1-\mathcal{O}\left(\Delta_{l}^{2}\right)$. Applying the recursive definitions for the $\hat{U}_{l}^{ \pm}$evolution operators [Eq. (2)],

$$
\hat{\Delta}_{l} \equiv \hat{U}_{l}^{+}-\hat{U}_{l}^{-}=\left[\hat{U}_{l-1}^{-}, \hat{U}_{l-1}^{+}\right]=\left[\hat{U}_{l-1}^{-}, \hat{\Delta}_{l-1}\right],
$$

noting that $\hat{U}_{l-1}^{-}$commutes with itself.

Consider a perturbation with a smallness parameter $\lambda$ in which $\hat{U}_{l}^{ \pm}=\mathbb{1}+\mathcal{O}(\lambda)$ for all $l \geq l_{0}$ for some $l_{0}$. Because the identity commutes with anything, Eq. (6) implies that $\hat{\Delta}_{l}=\mathcal{O}(\lambda) \times \hat{\Delta}_{l-1}$ for all $l>l_{0}$. A perturbation in the time parameter $\tau$ as well as a perturbation in the intrabath interactions $\epsilon$ both have this property with $l_{0}=0$ and $l_{0}=1$, respectively; CDD therefore cancels successive orders in $\tau$ and $\epsilon$. The successive cancellation in the time perturbation was previously noted in Refs. 10, but not that in the intrabath perturbation. 


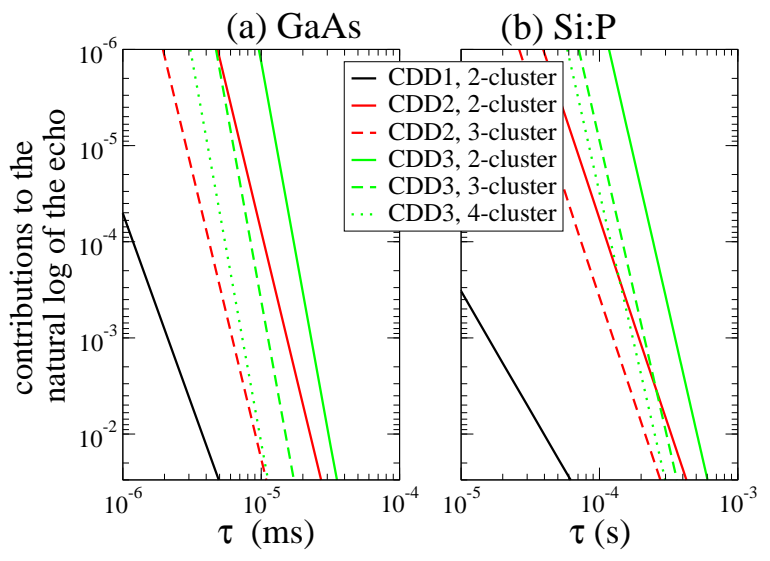

FIG. 3: Contributions from clusters of different sizes for the concatenated echo decays in the (a) Si:P system of Fig. 1 and (b) GaAs system of Fig. 2. The minimum cluster size required to yield the appropriate lowest-order result in the cluster expansion increases with each concatenation, and the larger clusters tend to dominate the decoherence.

Both of these perturbations are in correspondence with the cluster expansion because an increase in cluster size necessitates an increase in the minimum number of bilinear interaction factors $\epsilon$, and a corresponding increase in $\tau$ factors (by time-energy dimensional arguments). In fact, the cluster expansion converges due to the $\epsilon$ perturbation (the time perturbation is additionally applicable only in special cases) as demonstrated in Figs. 1 and 2 where the dotted lines give the results with $\left\langle\hat{W}^{[1]}\right\rangle$ approximated by the lowest order in $\epsilon$. This suggests that CDD provides relevant perturbative cancellations in the same regime where the cluster expansion converges.

With each perturbative cancellation, clusters of increasing size must be included in the lowest-order clus- ter expansion calculation as all have the same perturbative order. Contributions from different cluster sizes are shown for the CDD series in Fig. 3 for the Si:P and GaAs systems. The larger clusters of the same perturbative order are seen to dominate the decoherence because they are greater in number (concatenation of level $l$ is dominated by clusters of size $l+1$ ), invalidating the pair approximation used in Refs. 10. This is unfortunate since the pair approximation is simple and physically transparent.

In summary, we establish the effectiveness of CDD in correcting spectral-diffusion-induced quantum decoherence in the central spin problem. For our problem (and assuming ideal pulses), CDD effectively restores phase coherence when the interpulse delay is short compared with the typical intrabath interaction time scale. Furthermore, each level of concatenation involves larger clusters of bath spins, thus invalidating the simple pair approximation used earlier in the literature. In reality, the deviation from pulse ideality will determine the level of feasible quantum error correction; there is, however, promising recent work ${ }^{18}$ to address this issue.

Our study of spin bath decoherence provides important tests for CDD strategies and takes these strategies beyond the level of abstract formalism ${ }^{3,4}$ and small-scale models, $, 4,19$ establishing that CDD could play a key role in correcting decoherence-induced errors in solid-state spin quantum computer architectures. An ability to prolong quantum coherence indefinitely using CDD techniques could lead to an effective solid-state spin quantum memory as well as much reduced overhead in quantum error correction protocols.

We would like to acknowledge helpful comments from David Lidar and Kaveh Khodjasteh. This work is supported by DTO-ARO and NSA-LPS.
1 M. S. Byrd and D. A. Lidar, Phys. Rev. Lett. 89, 047901 (2002); Lian-Ao Wu, M.S. Byrd, and D.A. Lidar, ibid. 89, 127901 (2002).

${ }^{2}$ L. Viola, E. Knill, and S. Lloyd, Phys. Rev. Lett. 82, 2417 (1999);

3 L. Viola and S. Lloyd, Phys. Rev. A 58, 2733 (1998).

${ }^{4}$ K. Khodjasteh and D.A. Lidar, Phys. Rev. Lett. 95, 180501 (2005); Phys. Rev. A 75, 062310 (2007).

${ }^{5}$ L. Viola and E. Knill, Phys. Rev. Lett. 94, 060502 (2005).

6 O. Kern and G. Alber, Phys. Rev. Lett. 95, 250501 (2005).

7 W. Magnus, Commun. Pure Appl. Math. 7, 649 (1954).

8 H.Y. Carr and E.M. Purcell, Phys. Rev. 94, 630 (1954); S. Meiboom and D. Gill, Rev. Sci. Instrum. 29, 6881 (1958).

9 R. de Sousa and S. Das Sarma, Phys. Rev. B 68, 115322 (2003).

10 Wang Yao, Ren-Bao Liu, and L. J. Sham, Phys. Rev. Lett. 98, 077602 (2007); Ren-Bao Liu, Wang Yao, and L. J. Sham, New J. Phys. 9, 226 (2007).

11 D. Lidar (private communication).
12 B. Herzog and E.L. Hahn, Phys. Rev. 103, 148 (1956); A.M. Portis, ibid. 104, 584 (1956); J.R. Klauder and P.W. Anderson, ibid. 125, 912 (1962); C.P. Slichter, Principles of Magnetic Resonance, 3rd ed. (Springer-Verlag, Berlin, 1990).

13 W.M. Witzel, R. de Sousa, and S. Das Sarma, Phys. Rev. B 72, 161306(R) (2005).

14 W.M. Witzel and S. Das Sarma, Phys. Rev. B 74, 035322 (2006).

15 W.M. Witzel and S. Das Sarma, Phys. Rev. Lett. 98, 077601 (2007).

16 Wang Yao, Ren-Bao Liu, and L. J. Sham, Phys. Rev. B 74, 195301 (2006).

17 In terms of temperature, dipolar interactions have nK scale and nuclear Zeeman energies, at a field around $1 \mathrm{~T}$, have $\mathrm{mK}$ scale. The random bath assumption is therefore justified for typical thermal baths of $0.1-1 \mathrm{~K}$. Below the $\mathrm{mK}$ range, one may simply weight the average according to nuclear polarization. 
18 S. Pasini, T. Fischer, P. Karbach, and G. S. Uhrig, arXiv:0709.0588

19 Wenxian Zhang, V. V. Dobrovitski, Lea F. Santos, Lorenza
Viola, and B. N. Harmon, Phys. Rev. B 75, 201302(R) (2007). 